\title{
Performance Evaluation of Large Scaled Applications using Different Load Balancing Tactics in Cloud Computing
}

\author{
Minu Bala \\ Research Scholar \\ Department of C.S. \& I.T \\ University of Jammu, J\&K, India
}

\author{
Devanand, $\mathrm{PhD}$ \\ Professor \\ Department of C.S. \& I.T \\ University of Jammu, J\&K, India
}

\begin{abstract}
There is no doubt that workload distribution in cloud computing is one of the most important factors that regulates and dictates, directly or indirectly, its behavior and affects system performance. But geographical location of the datacenter and the user group is also an important factor that affects the overall performance of the system. This paper represents the performance analysis of three load balancing policies in combination with different broker policies for large-scaled applications using different infrastructural environments. The study has been made using CloudAnalyst: A CloudSim-based tool for modeling and analysis of large scale cloud computing environments. Experimental results reveal that large scaled software systems like FACEBOOK, TWITTER, ORKUT and e-commerce applications etc. can minimize their costs and improve service quality to the end users by making a right choice of service provider in the cloud market. The cloud service providers can also optimize their processing time by applying a best resource provisioning policy.
\end{abstract}

\section{Keywords}

Cloud Computing, IaaS, PaaS, SaaS, Load Balancing, Simulation.

\section{INTRODUCTION}

The rapid development of processing and storage technologies and the success of the internet has enabled the realization of new computing model called Cloud Computing[1], in which resources are provided as general utilities that can be leased and released by users through the internet in an on-demand fashion. Clouds offer services[2] that can be grouped into three categories: Software as a Service (SaaS), Platform as a Service (PaaS), and Infrastructure as a Service (IaaS).

Infrastructure as a Service: IaaS refers to on-demand provisioning of infrastructural resources, usually in terms of VMs. The cloud owner who offers IaaS is called an IaaS provider. Examples of IaaS providers include Amazon EC2, GoGrid etc.

Platform as a Service: PaaS refers to providing platform layer resources, including operating system support and software development frameworks. Examples of PaaS providers include Google App Engine, Microsoft Windows Azure etc.

Software as a Service: SaaS refers to providing on demand applications over the Internet. Examples of SaaS providers include Google Apps, Facebook etc.
With the advancement of the Cloud, there are new possibilities opening up on how applications can be built on the Internet. On one hand there are the cloud service providers who are willing to provide large scaled computing infrastructure at a cheaper price which is often defined on usage, eliminating the high initial cost of setting up an application deployment environment, and provide the infrastructure services in a very flexible manner which the users can scale up or down at will. On the other hand there are large scaled software systems such as social networking sites and e-commerce applications gaining popularity today which can benefit greatly by using such cloud services to minimize costs and improve service quality to the end users.

\section{FACTORS \\ AFFECTING \\ THE PERFORMANCE OF LARGE SCALED APPLICATIONS}

\subsection{Geographical Location}

The geographical location of service provider matters a lot in the overall performance of large scaled applications on the cloud due to different network issues and the location of the user groups. Amazon EC2[3] provides the ability to place instances in multiple locations. It is currently available in nine regions: USEast (Northern Virginia), USWest (Oregon), USWest (Northern California), EU (Ireland), Asia Pacific (Singapore), Asia Pacific (Tokyo), Asia Pacific ( Sydney), South America (Sao Paulo) and AWS GorCloud. By launching instances of an application from different regions, failure of an application from single region can be protected.

\subsection{Service Broker[4] Policy}

The Service Broker entity is responsible for routing users' requests coming from different user groups located at different geographical regions in globe to datacenters in cloud. The service broker policy can be of following types:

\subsubsection{Closest Datacenter Policy}

In this Policy, the Service Broker sends the request to closest datacenter in terms of Network Latency.

\subsubsection{Performance Optimization Policy}

In this policy, the service broker actively monitors all the datacenters and sends the request to the datacenter which gives best response time to the end user at the time it is queried. 


\subsubsection{Dynamic Configuration Policy}

In this policy, the service broker is assigned an additional job of scaling the application deployment depending upon the load it is currently facing. It increases or decreases VMs dynamically in the datacenters according to the current processing times as compared against the best processing times ever achieved.

\subsection{VM Load Balancing[5] Policy}

The Datacenter Controller (DCC) is an entity which uses the VM allocation policy to route the user requests, received in the form of cloudlet, to the VM for processing.

\subsubsection{Round Robin Policy}

In Round Robin Policy, the requests of the clients are handled in a circular manner on first come first bases. The DCC directly interacts with the users' requests and Load Balancer is the entity which assigns the load to the VMs. In this policy, the Load Balancer keeps record of all available VMs and also keeps track of next VM, to which next job is supposed to assign. As the new request comes from the user to DCC, it forwards request to the Load Balancer. The Load Balancer picks the next VM in circular order and assigns job in circular manner.

\subsubsection{Throttled Policy}

In this policy, each VM is assigned only one job at a time and another job can be assigned only when the current job has completed successfully. The Load Balancer entity maintains an index table of all VMs as well as their current states (Available or Busy). The client first makes a request to DCC for the allocation of appropriate $\mathrm{VM}$ and to perform the recommended job. The DCC queries the Load Balancer for allocation of the VM. The Load Balancer scans the index table from top to bottom until the first available VM is found. If it finds, returns the VM id to the DCC. Further, the DCC acknowledges Load Balancer about the new allocation and updates the index table of VM accordingly. On the other hand, if the Load Balancer doesn't find any VM in the available state, it simply returns null. In this case DCC queues the request until the availability of any VM. When a VM suitably finishes processing the request, it sends a signal to the DCC about the completion of a job. The DCC further sends a signal to Load Balancer about the de-allocation of VM and updating its status in the index table. The DCC sees, if there is any queued request, sends it to the Load Balance for further processing.

\subsubsection{Active Monitoring Policy}

In this policy, load is equally distributed among all the VMs by actively monitoring the load on all the VMs. The Load Balancer maintains an index table of virtual machines and the number of allocations assigned to each virtual machine. Upon the arrival of a new request, the DCC queries the Load Balancer for allocation of new VM. The Load Balancer parses the index table from top until the least loaded VM is found. When it finds, it returns the VM id to the DCC. If there is more than one found, it uses first come first serve (FCFS) basis to choose the least loaded. Simultaneously, it also returns the VM id to the DCC. The DCC notifies the Load Balancer about the new allocation. It updates the index table by increasing the allocation count by 1 for that VM. When a VM suitably finishes processing the assigned request, it forwards a response to the DCC. On receiving the response it notifies the Load Balancer about the VM de-allocation which further updates the index table by decreasing the allocation count for that VM by 1 .

\section{PROPOSED STUDY}

Quantifying the performance of different provisioning policies in a real cloud computing environment for different application models under variant conditions is extremely challenging due to the rigidity of the real infrastructure. Further, it is tedious and time consuming to reconfigure benchmarking parameters across massive scale cloud computing infrastructure over multiple test runs. So, the proposed study is to evaluate the performance of Large Scaled Application hosted at different geographical locations across the globe through simulations, using different resource provisioning policies.

\section{SIMULATION}

\subsection{Simulation Tool}

CloudAnalyst[6] is a GUI based simulator for modeling and analysis of large scaled applications. It is built on top of CloudSim toolkit, by extending CloudSim functionality with the introduction of concepts that model Internet and Internet Application behaviors.

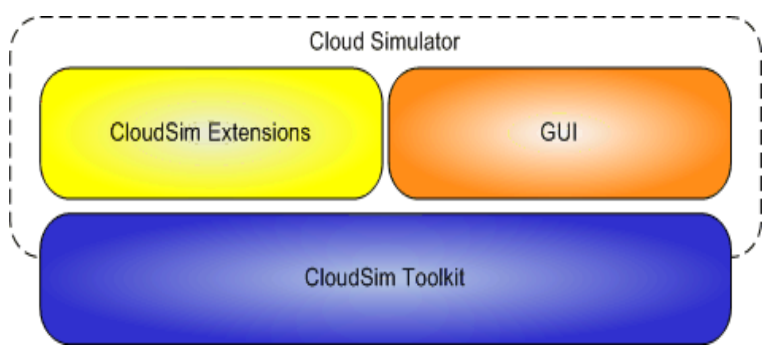

Fig 1: CloudAnalyst built on top of CloudSim toolkit

Through GUI, its different components like Userbase, Internet, Service Broker Policy, Internet Cloudlet, Datacenter Controller and VM Load Balancing Policies can be configured differently for different cloud scenarios.

\subsection{Simulation setup}

Large Scaled Applications that could be benefited from the cloud computing are Social Networking Applications, e-Commerce Applications, Online Education Applications etc. For the present study, a Social Networking Application namely Facebook has been considered. The approximate users of Facebook[7] distributed across the globe as on 31-03-2012 are as under:

Table 1. Registered users of FB as on 31-03-2012

\begin{tabular}{|c|c|}
\hline $\begin{array}{c}\text { Geographic Region in order of } \\
\text { size }\end{array}$ & Registered users \\
\hline Europe & $232,835,740$ \\
\hline Asia & $195,034,380$ \\
\hline North America & $173,284,940$ \\
\hline South America & 173284940 \\
\hline Central America & $41,332,940$ \\
\hline Africa & $40,205,580$ \\
\hline Middle East & 20,247900 \\
\hline Oceania/Australia & 13,597380 \\
\hline Caribbean,the & 6355320 \\
\hline
\end{tabular}


For the simulation purpose, the whole globe has been divided into six regions as R0, R1, R2, R3, R4 and R5. And grouping of registered users of FB as Userbases is done as under:

Table 2. Grouping of Regions \& Userbases

\begin{tabular}{|c|c|c|c|}
\hline Regions & $\begin{array}{c}\text { Names of } \\
\text { Geographic } \\
\text { Regions }\end{array}$ & Userbase & $\begin{array}{c}\text { Registered } \\
\text { users }\end{array}$ \\
\hline R2 & Europe & UB3 & 232835740 \\
\hline R3 & $\begin{array}{c}\text { Asia, Middle } \\
\text { East }\end{array}$ & UB4 & 215282280 \\
\hline R0 & $\begin{array}{c}\text { North America, } \\
\text { Central America, } \\
\text { Caribbean,the }\end{array}$ & UB1 & 220973200 \\
\hline R1 & South America & UB2 & 112531100 \\
\hline R4 & Africa & UB5 & 40205580 \\
\hline R5 & Oceania/Australia & UB6 & 13,597380 \\
\hline & & &
\end{tabular}

In order to bring the simulation framework more close to the real environment, the network behavior of the simulation model as been configured as per the network characteristics of Amazon EC2. It has been assumed that only 5\% of the registered users remain online during peak hours and $1 / 10^{\text {th }}$ of peak hour users will remain online during off-peak hours. It is further assumed that each user makes a new request after every 5 minutes when online. The following parameters have kept fixed for all simulation scenarios.

Table 3. Parameters fixed for simulations

\begin{tabular}{|c|c|}
\hline Parameter & Value \\
\hline Simulation duration & 60 min. \\
\hline Requests per user per hr. & 12 \\
\hline Data Size per request per hr. & 100 bytes \\
\hline $\begin{array}{c}\text { No. of Hosts (Each having } 4 \\
\text { processors) }\end{array}$ & 10000 \\
\hline $\begin{array}{c}\text { User Grouping factor in } \\
\text { Userbases }\end{array}$ & 1000 \\
\hline $\begin{array}{c}\text { Request Grouping factor in } \\
\text { Datacenters }\end{array}$ & 500 bytes \\
\hline $\begin{array}{c}\text { Executable instruction length } \\
\text { per request }\end{array}$ & 40 \\
\hline
\end{tabular}

\subsubsection{Scenario 1 Setup \& Observations}

In scenario 1, simulation runs have been made for one datacenter, by changing its location one by one to each geographical region, for three Service Broker Policies (Closet Data Center, Optimal Response Time and Dynamic Configuration) each in combination with three VM Load Balancing Policies (ESCE, RR, and Throttling) one by one. The observations made during all simulation runs are depicted in the following three tables.
Table 4. Overall Response Time of a datacenter using ESCE Load Balancing Policy

\begin{tabular}{c|c|c|c|}
\hline Regions & $\begin{array}{c}\text { Closest } \\
\text { Data } \\
\text { Center } \\
\text { Policy }\end{array}$ & $\begin{array}{c}\text { Optimal } \\
\text { Response } \\
\text { Time Policy }\end{array}$ & $\begin{array}{c}\text { Dynamic } \\
\text { Configuration } \\
\text { Policy }\end{array}$ \\
\hline R0 & 97187 & 97308 & 96674 \\
\hline R1 & 96972 & 96861 & 96583 \\
\hline R2 & 97250 & 97682 & 96623 \\
R3 & 97736 & 97410 & 97170 \\
R4 & 97099.7 & 97084.8 & 96409 \\
R5 & 97289 & 97215 & 96597 \\
\hline
\end{tabular}

Table 5. Overall Response Time of a datacenter using Round Robin Load Balancing Policy

\begin{tabular}{c|c|c|c|}
\hline Regions & $\begin{array}{l}\text { Closest } \\
\text { Data } \\
\text { Center } \\
\text { Policy }\end{array}$ & $\begin{array}{l}\text { Optimal } \\
\text { Response } \\
\text { Time Policy }\end{array}$ & $\begin{array}{l}\text { Dynamic } \\
\text { Configuration } \\
\text { Policy }\end{array}$ \\
\hline R0 & 101534 & 101160 & 100293 \\
\hline R1 & 100801 & 100782 & 99424 \\
\hline R2 & 101737 & 101928 & 100866 \\
R3 & 101064 & 101136 & 99840 \\
R4 & 101344 & 101118 & 99965 \\
\hline
\end{tabular}

Table 6. Overall Response Time of a datacenter using Throttled Load Balancing Policy

\begin{tabular}{c|c|c|c|}
\hline Regions & $\begin{array}{c}\text { Closest } \\
\text { Data } \\
\text { Center } \\
\text { Policy }\end{array}$ & $\begin{array}{c}\text { Optimal } \\
\text { Response } \\
\text { Time } \\
\text { Policy }\end{array}$ & $\begin{array}{c}\text { Dynamic } \\
\text { Configuration Policy }\end{array}$ \\
\hline R0 & 14063 & 14046 & 14057 \\
\hline R1 & 14229 & 14219 & 14211 \\
\hline R2 & 14081 & 14079 & 14052 \\
R3 & 14189 & 14187 & 14172 \\
R4 & 14341 & 14338 & 14319 \\
R5 & 14256 & 14266 & 14236 \\
\hline
\end{tabular}




\subsubsection{Scenario 2 Setup \& Observations}

In scenario 2, simulation runs have been made for two identical datacenters, by changing the location each datacenter one by one according to the combinations as (R0 \& R2), (R1 \& R2) and (R1 \& R3), for three Service Broker Policies (Closet Data Center, Optimal Response Time and Dynamic Configuration) each in combination with three VM Load Balancing Policies (ESCE, RR, and Throttling) one by one. The observations made during all simulation runs are depicted in the following three tables:

Table 7. Overall Response Time of two datacenters using ESCE Load Balancing Policy

\begin{tabular}{c|c|c|c|}
\hline Regions & $\begin{array}{c}\text { Closest Data } \\
\text { Center Policy }\end{array}$ & $\begin{array}{c}\text { Optimal } \\
\text { Response } \\
\text { Time Policy }\end{array}$ & $\begin{array}{c}\text { Dynamic } \\
\text { Configuration } \\
\text { Policy }\end{array}$ \\
\hline R0 \& R2 & 57823.3 & 56307.73 & 55704.27 \\
\hline R1 \& R2 & 57815.65 & 54592.58 & 55697.57 \\
\hline R1 \& R3 & 56468.52 & 53461.12 & 54498.55 \\
\hline
\end{tabular}

Table 8. Overall Response Time of two datacenters using Round Robin Load Balancing Policy

\begin{tabular}{c|c|c|c|}
\hline Regions & $\begin{array}{c}\text { Closest Data } \\
\text { Center Policy }\end{array}$ & $\begin{array}{c}\text { Optimal } \\
\text { Response } \\
\text { Time Policy }\end{array}$ & $\begin{array}{c}\text { Dynamic } \\
\text { Configuration } \\
\text { Policy }\end{array}$ \\
\hline R0 \& R2 & 59171.72 & 50960.86 & 56894.81 \\
\hline R1 \& R2 & 58870.71 & 55432.02 & 56828.59 \\
\hline R1 \& R3 & 56678.21 & 52752.64 & 54130.9
\end{tabular}

Table 9. Overall Response Time of two datacenters using Throttled Load Balancing Policy

\begin{tabular}{c|c|c|c|}
\hline Regions & $\begin{array}{c}\text { Closest Data } \\
\text { Center Policy }\end{array}$ & $\begin{array}{c}\text { Optimal } \\
\text { Response } \\
\text { Time Policy }\end{array}$ & $\begin{array}{c}\text { Dynamic } \\
\text { Configuration } \\
\text { Policy }\end{array}$ \\
\hline R0 \& R2 & 10177.81 & 9532.12 & 10157.8 \\
\hline R1 \& R2 & 10203.52 & 9638.43 & 10179.89 \\
\hline R1 \& R3 & 10097.06 & 9323.4 & 11205.87
\end{tabular}

4.3 Graphical Representation and Analysis The graphical representation of experimental observations obtained in scenario 1 are depicted in Fig. 2, 3, 4 \& 5.

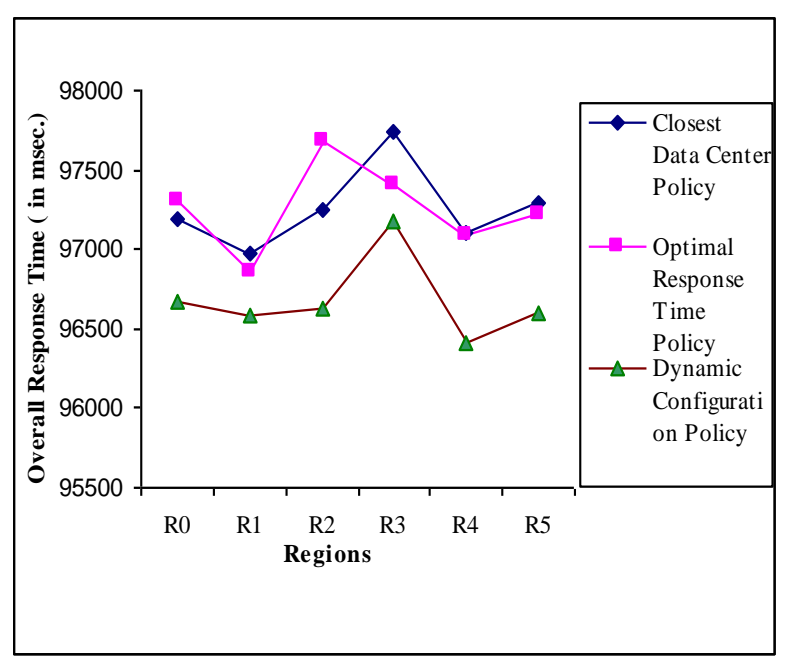

Fig.2 Overall Response Time of a Datacenter at different regions using different Policies at Broker Level and Active Monitoring LB Policy at VM level

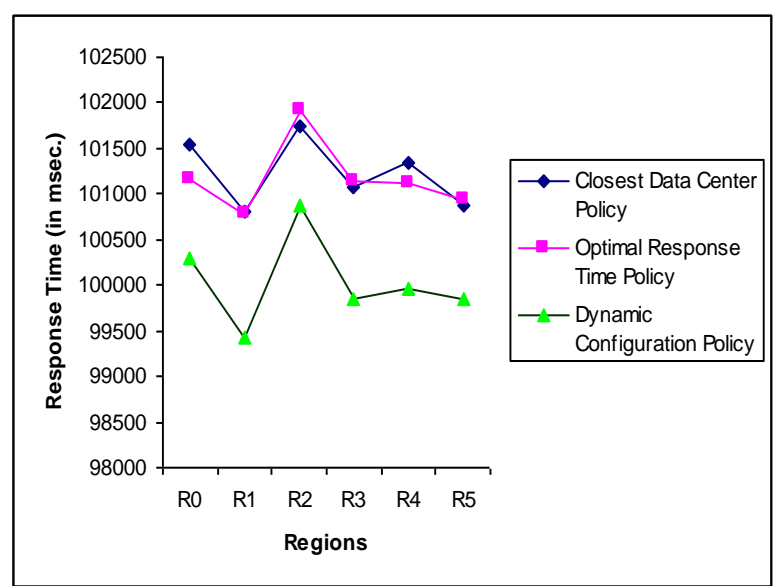

Fig.3 Overall Response Time of a Datacenter at different regions using different Policies at Broker Level and Round Robin LB Policy at VM level

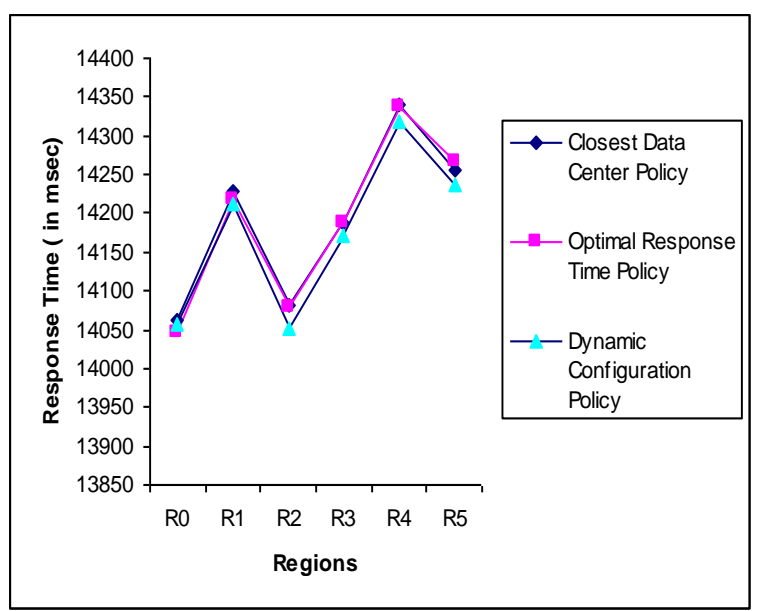

Fig.4 Overall Response Time of a Datacenter at different regions using different Policies at Broker Level and Throttled LB Policy at VM level 


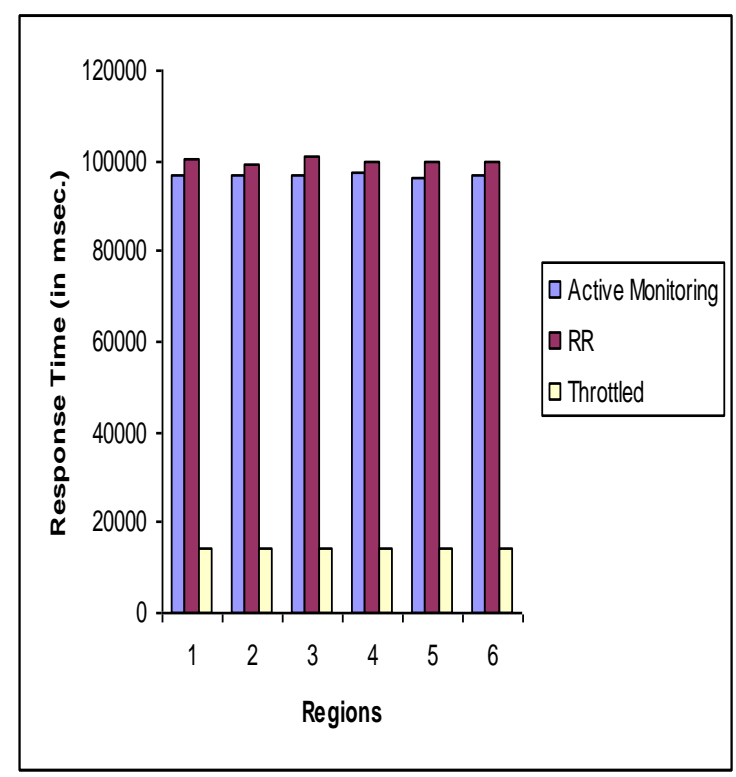

Fig. 5 Overall Response Time of a Datacenter at different regions using Dynamic Configuration Policy at Broker

Level and different LB Policies at VM Level

The experimental results depicted in Fig. 2, Fig. 3 and Fig. 4 reveals that the overall response time of a datacenter changes with the change in the geographical location of datacenter. It is better when the service broker policy is Dynamic than other two policies (Closest Data Center, Optimal Response Time) using any of the three VM Load Balancing Policies considered in the experimentation. The result analysis in Fig. 5 reveals that out of the three considered VM Load Balancing Policies, the overall response time of the Datacenter is better in case of Throttled Load balancing Policy. And the results are better when the geographical location of the datacenter is Region R2 than other five regions.

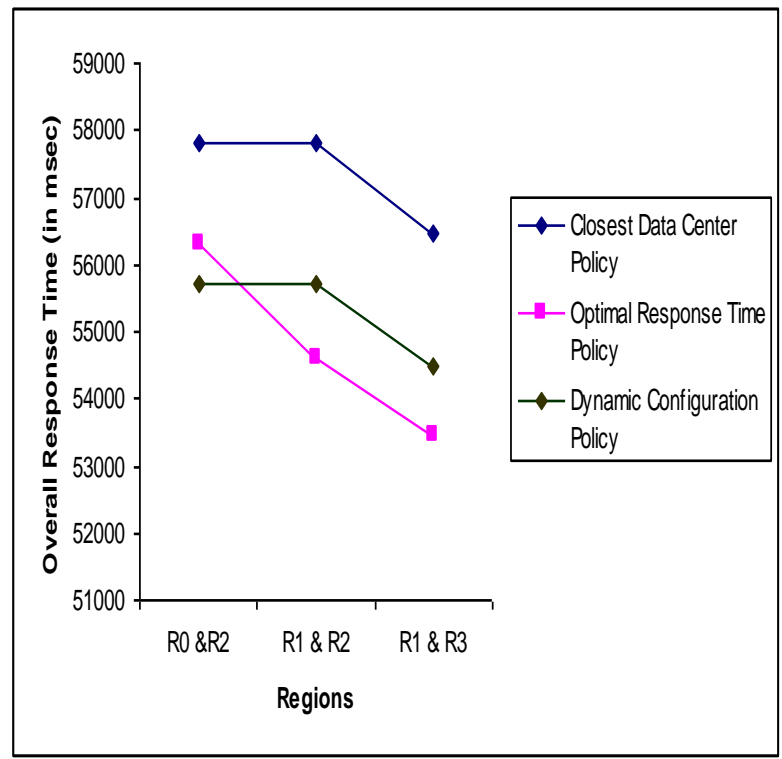

Fig.6 Overall Response Time of two Datacenters located at two different regions using different policies at broker level and Active Monitoring LB Policy at VM level

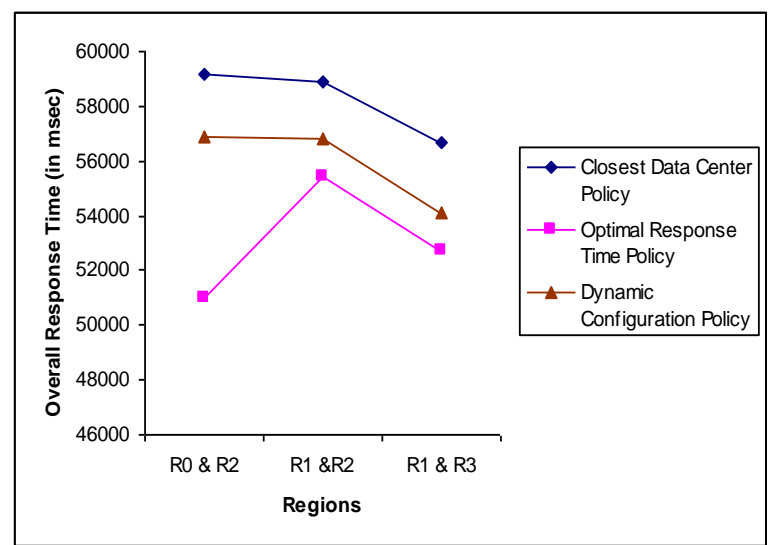

Fig.7 Overall Response Time of two Datacenters located at two different regions using different policies at broker level and Round Robin LB Policy at VM level

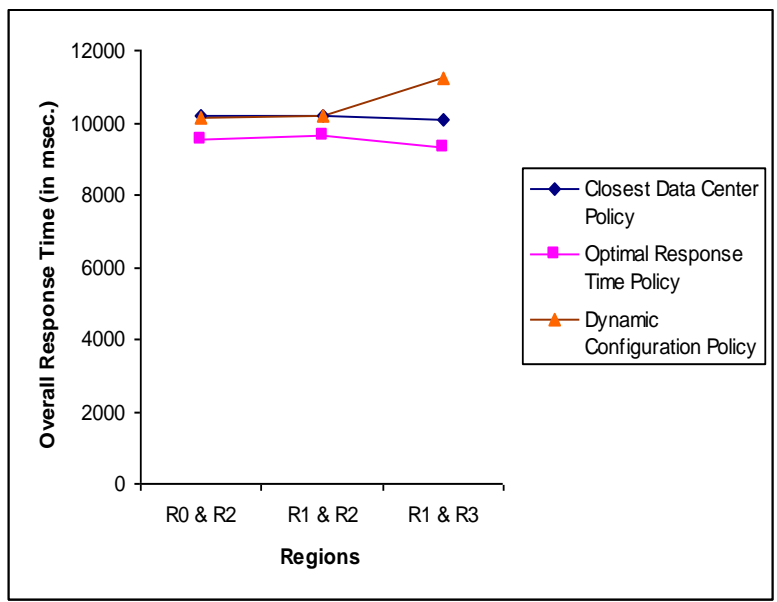

Fig.8 Overall Response Time of two Datacenters located at two different regions using different policies at broker level and Throttled LB Policy at VM level

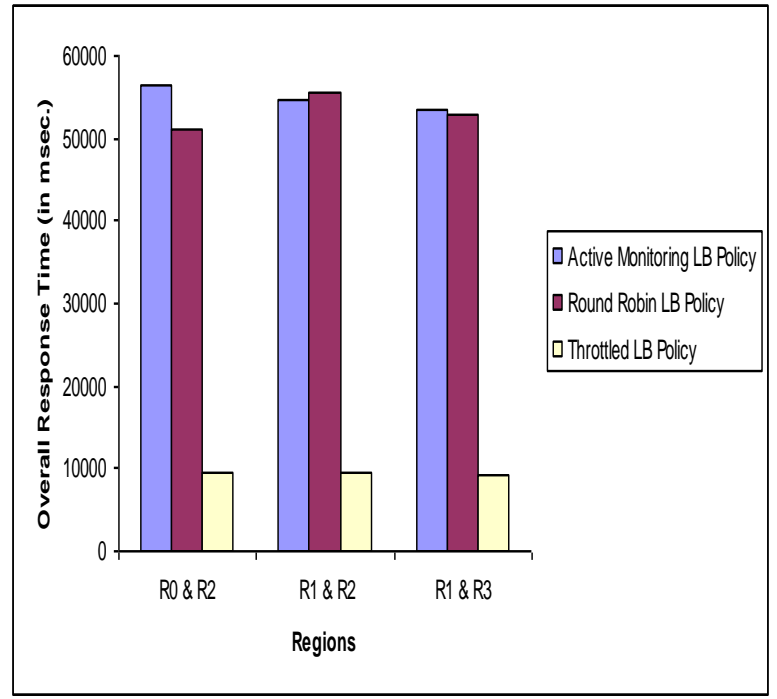

Fig.9 Overall Response Time of two Datacenters located at two different regions using Optimize Response Time Policy at broker level and different LB Policies at VM Level 


\section{CONCLUSION}

Besides service broker policy and VM load balancing policy, the geographical location of the datacenter where a large scale application is hosted and the geographical location of its end users, affect the overall performance of the system. The choice of datacenter's location according to location of its maximum end users improves the results. Thus the overall response time of the application to the end users can be optimized by making a right combination of the above mentioned elements. Moreover such type of simulation work helps to generate a valuable insight for Application Designers in identifying the optimal configuration for their application.

\section{REFERENCES}

[1] Luis M. Vaquero, Luis Rodero-Merino, Juan Caceres, Maik Lindner, "A Break in the Clouds: Towards a Cloud Definition", ACM SIGCOMM Computer Communication Review, Volume 39, Number 1, Pages 50-55, Jan. 2009.

[2] Rajkumar Buyya, Chee Shin Yeo, Srikumar Venugopal, James Broberg, and Ivona Brandic, "Cloud Computing and Emerging IT Platforms: Vision, Hype, and Reality for Delivering Computing as the 5th Utility, Future
Generation Computer Systems", Volume 25, Number 6, Pages: 599-616, ISSN: 0167-739X, Elsevier Science, Amsterdam, The Netherlands, June 2009.

[3] www.aws.amazon.com/ec2

[4] R. Buyya, R. Ranjan, and R. N. Calheiros, "Modeling And Simulation Of Scalable Cloud Computing Environments And The Cloudsim Toolkit: Challenges And Opportunities," Proc. Of The 7th High Performance Computing and Simulation Conference (HPCS 09), IEEE Computer Society, June 2009.

[5] Jasmin James and Dr. Bhupendra Verma, "Efficient VM load balancing algorithm for a cloud computing environment", International Journal on Computer Science and Engineering (IJCSE), 09 Sep 2012.

[6] Bhathiya Wickremasinghe, "CloudAnalyst: A CloudSim based Tool for Modeling and Analysis of Large Scale Cloud Computing Environments" MEDC project report, 433-659 Distributed Computing project, CSSE department., University of Melbourne, 2009.

[7] www.internetworldststs.com/facebook.htm 